\title{
How stationarity contradicts intergenerational equity
}

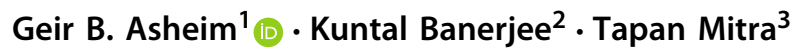

Received: 7 April 2020 / Accepted: 8 July 2020 / Published online: 17 September 2020

(๑) The Author(s) 2020

\begin{abstract}
We show how the condition of stationarity may contradict intergenerational equity. By formalizing the intuition that less sensitivity remains for the continuation of the stream if sensitivity for the interests of the present is combined with stationarity, we point out conflicts (a) between stationarity and the requirement of not letting the present be dictatorial, and (b) between stationarity and equal treatment of generations. We use the results to interpret the non-stationarity of the Chichilnisky and Rankdiscounted utilitarian social welfare functions. Non-stationarity combined with time invariance leads to time inconsistency. We illustrate how such non-stationary social welfare functions can be applied in the Ramsey model if time invariance is imposed.
\end{abstract}

Keywords Intergenerational equity $\cdot$ Stationarity $\cdot$ Time inconsistency

JEL Classification D63 · D71 · Q01

\begin{abstract}
Tapan Mitra passed away on 3 February 2019. Through his work he seamlessly combined authority and grace. He remains a valuable source of inspiration to his co-authors. We thank two anonymous referees, Walter Bossert, Claude d'Aspremont, Marc Fleurbaey, Sean Horan, Adam Jonsson, Paolo Piacquadio, and other seminar participants in Lund, Marseille, Montréal, Oslo, Taipei, Tokyo, and Tucson for helpful comments. This paper is part of the research activities at the Centre for the Study of Equality, Social Organization, and Performance (ESOP) at the Department of Economics at the University of Oslo. Asheim's research has benefitted from stays at IMéRA-Marseille, CIREQ-Montréal, and Paris School of Economics. An earlier version of this paper was circulated under the title "The necessity of time inconsistency for intergenerational equity".
\end{abstract}

$\bowtie \quad$ Geir B. Asheim

g.b.asheim@econ.uio.no

Kuntal Banerjee

kbanerje@fau.edu

Tapan Mitra

tm19@cornell.edu

1 Department of Economics, University of Oslo, P.O. Box 1095, Blindern 0317, Oslo, Norway

2 Department of Economics, Florida Atlantic University, 777 Glades Road, Boca Raton, FL 33431, USA

3 Department of Economics, Cornell University, 448 Uris Hall, Ithaca, NY 14853, USA 


\section{Introduction}

Intergenerational equity is often studied in a context where the time horizon is infinite. This modeling choice captures that there is a large number of future potential people by distributing them over a countably infinite number of generations. If we assume that the wellbeing of each of these generations can be measured by a level-comparable index, then the question of intergenerational equity corresponds to the problem of ranking such infinite wellbeing streams by means of a social welfare relation.

Following Koopmans (1960, Section 6), a social welfare relation satisfies the property of stationarity if for any two streams with the same present wellbeing, the social preference between these stream is not changed if the timing of the future components of the streams are advanced one period and one forgets about the common present component. Stationarity is a key axiom in Koopmans's (1960) axiomatization of the time-discounted utilitarian social welfare function whereby two streams are compared by the discounted sum of future transformed wellbeing, with a constant positive perperiod discount factor smaller than 1 .

If a social welfare relation is stationary, then it can be both time consistent and time invariant. Otherwise, one must confront the dilemma of choosing between time consistency and time invariance, so that time consistency can be satisfied only if the social welfare relation deviates from consequentialism in terms of the future properties of the stream by being time variant. During the last 25 years there has been a large interest in the positive study of non-stationary individual preferences, independently of whether the non-stationarity manifests itself as time inconsistency or time variance or both (Halevy 2015). In this paper we make a more novel claim by arguing that non-stationarity is important in a normative context, namely for the social evaluation of intergenerational wellbeing streams.

There is a basic intuition for why stationarity might be in conflict with equitable treatment of generations: if sensitivity for the interests of the present is combined with stationarity, then less sensitivity remains for the continuation of the stream. This has two consequences:

- Impatience, thereby contradicting equal treatment in the sense of anonymity.

- No weight remains in the limit as time goes to infinity for the tail of the stream under sufficient continuity, leading to dictatorship of the present.

This intuition will be developed in the present paper.

Intertemporal social choice theory yields axiomatic foundation for criteria of intergenerational equity. As we explore in Sect. 2, such axioms can be divided into four classes:

(1) Imposing coherency, richness, and robustness through axioms like numerical representability and continuity.

(2) Sensitivity axioms, like different variants of the Pareto principle, specifying when added wellbeing leads to a better stream. The strong Pareto principle entails that adding wellbeing always improves the stream.

(3) Equity axioms, like anonymity or non-dictatorship of the present.

(4) Separability axioms, including stationarity. 
Throughout we impose representability by means of a social welfare function, ensuring transitivity (as a coherency property) and completeness (as a richness property), as well as monotonicity in the sense that adding wellbeing can never lead to a worse stream. We provide results on how requiring stationarity leads to conflicts with equity axioms, under continuity and sensitivity axioms.

A social welfare function satisfying continuity and the strong Pareto principle cannot both be stationary and satisfy non-dictatorship of the present on the set of converging streams (Proposition 1 of Sect. 3). Hence, the ranking of two converging streams depends only on the properties of the streams before some finite time $\tau$, and will not change even if, beyond time $\tau$, the less preferred stream is changed to a stream leading to bliss and the more preferred stream is changed to one leading to destitution. We show how this result is tight by demonstrating possibility if any one axiom is removed. In particular, Chichilnisky (1996) provides a class of social welfare functions which respect continuity, the strong Pareto principle, and non-dictatorship of the present, but they do not satisfy stationarity. Moreover, two examples illustrate how the dilemma can be resolved by dropping continuity. However, as discussed at the end of Sect. 3, we have not been able to construct an "attractive" social welfare function that combines the strong Pareto principle, stationarity, and non-dictatorship of the present on the set of converging streams.

Under the strong Pareto principle, a social welfare function cannot be anonymous, as shown by Basu and Mitra (2003). So there is an impossibility even before imposing stationarity. However, a monotone social welfare function can satisfy anonymity by weakening the strong Pareto principle to restricted dominance. Restricted dominance means that a stream is made worse by reducing the wellbeing of the present generation if the starting point is an egalitarian stream. A monotone social welfare function satisfying restricted dominance as well as a very weak continuity axiom cannot both be stationary and anonymous (Proposition 2 of Sect. 4). We demonstrate by means of two examples how the dilemma can be resolved by weakening sensitivity even further, for example, by letting evaluation depend solely on the limit of streams, thus having the unattractive feature of not being sensitive to any finite part of streams. In Zuber and Asheim's (2012) axiomatization of Rank-discounted utilitarian social welfare function, stationarity holds only on the set of nondecreasing streams. Therefore, while the Rank-discounted utilitarian social welfare function is monotone and continuous and satisfies restricted dominance and anonymity, it might fail stationarity when comparing streams that are not nondecreasing.

We discuss the dilemma between time consistency and time invariance that nonstationarity poses in Sect. 5, in the context of the Chichilnisky and Rank-discounted utilitarian social welfare functions. If we insist on consequentialism in terms of the future properties of the stream by imposing time invariance, the resulting time inconsistency turns models of economic growth into intergenerational games. We illustrate such game-theoretic analysis by applying the Chichilnisky and Rank-discounted utilitarian social welfare functions to the Ramsey model. We discuss in Sect. 6 to what extent stationarity is a requisite property in rational social decision-making. Finally, we prove Propositions 1 and 2 in Appendix A and verify that the Chichilnisky and Rank-discounted utilitarian social welfare functions are not stationary in Appendix B. 
This paper follows in the tradition of a literature seeking to investigate the limits of possibility when evaluating infinite wellbeing streams. Like Diamond (1965), Basu and Mitra (2003), Crespo et al. (2009), and Alcantud (2012), we face the roadbloc of impossibility when trying to reconcile sensitivity and equity with representability and continuity. Our emphasis is different, though, as we highlight the particular role that stationarity plays. We are not aware of previous discussions in the literature of the conflict between stationarity and the requirement of not letting the present be dictatorial. However, we are not the first to point out a conflict between stationarity and equal treatment. In the setting of a sensitive social welfare function, Koopmans (1960) shows through his Theorem 1 how impatience is implied by stationarity. In fact, this is the main result of his seminal article, and the implication from stationarity to impatience is reflected by its title. More recently, Dutta (2008, Proposition 2) shows that if the alternating streams $(1,0,1,0,1,0, \ldots)$ and $(0,1,0,1,0,1, \ldots)$ are comparable under the strong Pareto principle and stationarity, then the former stream is strictly preferred to the latter, thereby exhibiting a weak form of impatience (see also Jonsson and Voorneveld 2015, Proposition 1, not included in the published version Jonsson and Voorneveld 2018). In the present paper we show through our Proposition 2 how stationarity leads to impatience also in Koopmans' (1960) stronger sense even under conditions that would otherwise be consistent with anonymity.

\section{Framework and axioms}

Denote by $\mathbb{N}$ the set of all positive integers and by $\mathbb{R}$ the set of all real numbers. Let, for all $t \in \mathbb{N}, x_{t} \in \mathbb{R}$ be an indicator of the wellbeing of generation $t$, and let $\mathbf{x}=\left(x_{1}, x_{2}, \ldots, x_{t}, \ldots\right)$ be an infinite wellbeing stream. A wellbeing stream $\mathbf{x}$ is constant if there is $a \in \mathbb{R}$ such that $x_{t}=a$ for all $t \in \mathbb{N}$, and we write $\mathbf{x}=a_{\text {con }}$. A wellbeing stream $\mathbf{x}$ is converging if $\lim _{t \rightarrow \infty} x_{t}$ exists. We normalize wellbeing to lie within the unit interval, $[0,1]$. This normalization is common in the literature on intergenerational equity and leads to little loss of generality. Write $\mathbf{X}=[0,1]^{|\mathbb{N}|}$ for the set of possible wellbeing streams and $\mathbf{X}^{c}$ for the subset of streams that are converging. For all $\mathbf{x}, \mathbf{y} \in \mathbf{X}$, write $\mathbf{x} \geq \mathbf{y}$ whenever $x_{t} \geq y_{t}$ for all $t \in \mathbb{N}$, and write $\mathbf{x}>\mathbf{y}$ if $\mathbf{x} \geq \mathbf{y}$ and $\mathbf{x} \neq \mathbf{y}$.

We study a social welfare relation (SWR) $\succsim$ that ranks elements of $\mathbf{X}$ (or $\mathbf{X}^{c}$ ). We interpret the SWR $\succsim$ as the social ranking made at time 1 over streams starting at time 1 . We say that a social welfare function (SWF) $w: \mathbf{X} \rightarrow \mathbb{R}\left(\right.$ or $\left.w: \mathbf{X}^{c} \rightarrow \mathbb{R}\right)$ represents a SWR $\succsim$ if, for all $\mathbf{x}, \mathbf{y} \in \mathbf{X}$ (or $\left.\mathbf{X}^{c}\right), \mathbf{x} \succsim \mathbf{y}$ if and only if $w(\mathbf{x}) \geq w(\mathbf{y})$. Throughout we consider a SWR $\succsim$ that is representable, implying that $\succsim$ is reflexive, transitive, and complete. Furthermore, throughout we impose that the representing SWF $w$ is monotone in the sense that $\mathbf{x}>\mathbf{y}$ implies $w(\mathbf{x}) \geq w(\mathbf{y})$. Such monotonicity is a weak minimal requirement, as it is does not rule out trivial preference in the sense that $w(\mathbf{x})=w(\mathbf{y})$ for all $\mathbf{x}, \mathbf{y} \in \mathbf{X}$ (or $\mathbf{X}^{c}$ ).

The axioms that we will consider fall into four classes (where $\mathbf{X}^{c}$ substitutes for $\mathbf{X}$ for a SWF with domain $\mathbf{X}^{c}$ ). One class consists of two continuity axioms that impose varying degrees of robustness of the SWF $w$. The first is regular continuity when we 
endow $\mathbf{X}$ with the supremum metric $d$ which, for any $\mathbf{x}, \mathbf{y} \in \mathbf{X}$, determines the distance $d(\mathbf{x}, \mathbf{y})=\sup \left\{\left|x_{t}-y_{t}\right|: t \in \mathbb{N}\right\}$.

Continuity The SWF $w$ is continuous in the metric space $(\mathbf{X}, d)$.

The second is a very weak continuity axiom on the diagonal of $\mathbf{X}$, that is, on the subset of constant streams. For its statement, define $f_{w}:[0,1] \rightarrow \mathbb{R}$ by $f_{w}(a)=w\left(a_{\text {con }}\right)$, and let $D\left(f_{w}\right) \subseteq[0,1]$ be the subset of the domain of $f_{w}$ at which $f_{w}$ is discontinuous.

Limited discontinuity The set $D\left(f_{w}\right)$ is not dense in $[0,1]$.

Clearly, Continuity implies Limited discontinuity since $D\left(f_{w}\right)=\emptyset$ if $w$ satisfies the former axiom.

The next class are sensitivity axioms. The strong Pareto principle belongs here:

Strong Pareto For all $\mathbf{x}, \mathbf{y} \in \mathbf{X}, \mathbf{x}>\mathbf{y}$ implies $w(\mathbf{x})>w(\mathbf{y})$.

Strong Pareto implies the following axiom, which requires sensitivity when moving away from the diagonal by reducing the first component.

Restricted dominance For all $a, b \in[0,1], a<b$ implies $w\left(a, b_{\text {con }}\right)<w\left(b_{\text {con }}\right)$.

Restricted dominance ensures some sensitivity to the interests of the present generation and implies Strong Pareto on the diagonal:

$$
\text { For all } a, b \in[0,1], a<b \text { implies } w\left(a_{\text {con }}\right) \leq w\left(a, b_{\text {con }}\right)<w\left(b_{\text {con }}\right)
$$

since $w$ is monotone. Hence, Restricted dominance combined with Continuity implies that there exists $w: \mathbf{X} \rightarrow[0,1]$ representing $\succsim$ with the property that $w\left(c_{\text {con }}\right)=c$ for all $c \in[0,1]$. To see this, let $v$ be a monotone SWF satisfying Restricted dominance and Continuity, implying that $f_{v}$ is continuous. By (1), $f_{v}$ is increasing so that the inverse $f_{v}^{-1}$ of $f_{v}$ exists and is increasing on $f_{v}([0,1])$. By continuity of $f_{v}$ and the intermediate value theorem, $f_{v}([0,1])$ equals the interval $\left[v\left(0_{\text {con }}\right), v\left(1_{\text {con }}\right)\right]$ so that, by monotonicity, the range of $f_{v}$ equals the range of $v$. Thus, the function $w=f_{v}^{-1} \circ v$ is a well-defined representation of $\succsim$ and has the property that $w\left(c_{\text {con }}\right)=c$ for all $c \in[0,1] .{ }^{1}$ Also, $w$ with this property is unique. To see this, suppose both $w^{\prime}, w^{\prime \prime}$ represent $\succsim$ and have the desired property. Then, for any $\mathbf{x} \in \mathbf{X}, w^{\prime}(\mathbf{x})=a=w^{\prime}\left(a_{\mathrm{con}}\right)$ and $w^{\prime \prime}(\mathbf{x})=b=w^{\prime \prime}\left(b_{\text {con }}\right)$ for some $a, b \in[0,1]$. Hence, $a_{\text {con }} \sim \mathbf{x} \sim b_{\text {con }}$ and, by $(1), w^{\prime}(\mathbf{x})=a=b=w^{\prime \prime}(\mathbf{x})$.

The time-discounted utilitarian (TDU) SWF $w_{\beta}^{T}: \mathbf{X} \rightarrow[0,1]$ belongs to this class:

$$
w_{\beta}^{T}(\mathbf{x})=u^{-1}\left[(1-\beta) \sum_{t=1}^{\infty} \beta^{t-1} u\left(x_{t}\right)\right],
$$

where $u:[0,1] \rightarrow \mathbb{R}$ is an increasing and continuous function that transforms wellbeing into generalized utility, and $\beta \in(0,1)$ is the utility discount rate. By multiplying with $(1-\beta)$, one obtains average discounted generalized utility and, by using the inverse $u^{-1}$, average discounted generalized utility is mapped into [0,1].

\footnotetext{
${ }_{1}^{1}$ Without Continuity the existence of a SWF $w$ with the property that $w\left(c_{\operatorname{con}}\right)=c$ for all $c \in[0,1]$ is not ensured. Example 1 of Sect. 3 illustrates by means of a counterexample why Continuity is needed.
} 
The third class consists of equity axioms. The first imposes impartiality by imposing invariance to finite permutations. A permutation $\pi$ of $\mathbb{N}$ is a bijection on $\mathbb{N}$. A permutation $\pi$ is finite if there exists $\tau \in \mathbb{N}$ such that $\pi(t)=t$ for all $t \geq \tau$. For all $\mathbf{x} \in \mathbf{X}$, write $\mathbf{x}_{\pi}=\left(x_{\pi(1)}, x_{\pi(2)}, \ldots\right) \in \mathbf{X}$ for the stream permuted by $\pi$.

Finite anonymity For all $\mathbf{x} \in \mathbf{X}$, if $\pi$ is a finite permutation, then $w(\mathbf{x})=w\left(\mathbf{x}_{\pi}\right)$.

It follows from Basu and Mitra (2003) that Strong Pareto is incompatible with Finite anonymity under our assumption that $\succsim$ is representable.

Chichilnisky (1996) says that there is dictatorship of the present if only what happens before a finite time matters for the ranking of alternatives. That is, for all $\mathbf{x}, \mathbf{y} \in \mathbf{X}$ with $w(\mathbf{x})>w(\mathbf{y})$, there exists $\tau \in \mathbb{N}$ such that

$$
w\left(x_{1}, \ldots, x_{t}, u_{t+1}, \ldots\right)>w\left(y_{1}, \ldots, y_{t}, v_{t+1}, \ldots\right)
$$

for all $t \geq \tau$ and $\mathbf{u}, \mathbf{v} \in \mathbf{X}$. In particular, for all $\mathbf{x}, \mathbf{y} \in \mathbf{X}$ with $w(\mathbf{x})>w(\mathbf{y})$, there exists $\tau \in \mathbb{N}$ such that $w\left(x_{1}, \ldots, x_{\tau}, 0_{\text {con }}\right)>w\left(y_{1}, \ldots, y_{\tau}, 1_{\text {con }}\right)$. Thus, the ranking of two streams depends only on the properties of the streams before some finite time $\tau$, and will not change even if, beyond time $\tau$, the more preferred stream is changed to one leading to destitution and the less preferred stream is changed to one leading to bliss. The ethically commendable property is that there is no dictatorship of the present, leading to the following axiom, which we strengthen by requiring the existence of converging streams $\mathbf{x}$ and $\mathbf{y}$ that contradict dictatorship of the present.

Non-dictatorship of the present (on the set of converging streams) There exist $\mathbf{x}$, $\mathbf{y} \in \mathbf{X}^{c}$ with $w(\mathbf{x})>w(\mathbf{y})$ such that, for all $\tau \in \mathbb{N}$, there exist $t \geq \tau$ and $\mathbf{u}, \mathbf{v} \in \mathbf{X}$ such that $w\left(x_{1}, \ldots, x_{t}, u_{t+1}, \ldots\right) \leq w\left(y_{1}, \ldots, y_{t}, v_{t+1}, \ldots\right)$.

The fourth and last class consists of separability axioms, where the following plays a key role in our analysis.

Stationarity For all $\mathbf{x}, \mathbf{y} \in \mathbf{X}$ with $x_{1}=y_{1}, w(\mathbf{x}) \geq w(\mathbf{y})$ if and only if $w\left(\mathbf{x}^{\prime}\right) \geq w\left(\mathbf{y}^{\prime}\right)$, where $x_{t}^{\prime}=x_{t+1}$ and $y_{t}^{\prime}=y_{t+1}$ for all $t \in \mathbb{N}$.

Stationarity is the conjunction of Postulates 3b and 4 in Koopmans' (1960) characterization of time-discounted utilitarianism. ${ }^{2}$ We have that Restricted dominance combined with Stationarity implies sensitivity for the future:

$$
\text { For any } a, b, c \in[0,1], \quad b<c \text { implies } w\left(a, b_{\mathrm{con}}\right)<w\left(a, c_{\mathrm{con}}\right) \text {. }
$$

This follows by applying Stationarity to (1), which is implied by Restricted dominance. Adding Continuity, we can define the aggregator function $g:[0,1]^{2} \rightarrow[0,1]$ by:

$$
g(a, b)=w\left(a, b_{\text {con }}\right)
$$

\footnotetext{
2 This terminology is used by, among others, Halevy (2015) and Millner and Heal (2018). The axiom was introduced (in a slightly stronger form) by Fleurbaey and Michel (2003); they called it Independent future. Koopmans (1960) used the term 'stationarity' when introducing his Postulate 4.
} 
where $w$ has the property that $w\left(c_{\mathrm{con}}\right)=c$ for all $c \in[0,1]$. For all $\mathbf{x} \in \mathbf{X}$, denote $w\left(\mathbf{x}^{\prime}\right)$ by $b$, where $x_{t}^{\prime}=x_{t+1}$ for all $t \in \mathbb{N}$. Hence, $w\left(\mathbf{x}^{\prime}\right)=b=w\left(b_{\text {con }}\right)$, and it follows by Stationarity that

$$
w(\mathbf{x})=w\left(x_{1}, x_{2}, \ldots\right)=w\left(x_{1}, b_{\text {con }}\right)=g\left(x_{1}, b\right)=g\left(x_{1}, w\left(\mathbf{x}^{\prime}\right)\right) .
$$

Under Continuity, Restricted dominance and Stationarity, $g$ satisfies:

(G.1) $g(a, b)<b$ if $a<b$ and $g(a, b)=b$ if $a=b$;

(G. $\left.2^{\prime}\right) g(a, b)$ is nondecreasing in $a$ given $b$;

(G.3) $g(a, b)$ is increasing in $b$ given $a$;

(G.4) $g(a, b)$ is continuous in $(a, b)$ on $[0,1]^{2}$.

If Strong Pareto replaces Restricted dominance, then $\left(\mathrm{G} .2^{\prime}\right)$ is strengthened to:

(G.2) $g(a, b)$ is increasing in $a$ given $b$.

\section{Stationarity leads to dictatorship of the present}

The main result of the present section is to demonstrate that the combination of Continuity, Strong Pareto, and Stationarity contradicts Non-dictatorship of the present (on the set of converging streams). Hence, if we insist that a monotone SWF $w$ satisfy Nondictatorship of the present (on the set of converging streams) as well as robustness in the sense of Continuity and sensitivity in the sense of Strong Pareto, then $w$ cannot be stationary. To motivate the analysis, we first present a weaker version of the result, where we only consider constant streams.

Observation 1 Assume that the monotone SWF $w$ satisfies Continuity, Strong Pareto, and Stationarity. For all $a, b \in[0,1]$, if $a<b$, then there exists $\tau \in \mathbb{N}$ such that, for every $t \geq \tau$,

$$
w(\underbrace{a, \ldots, a}_{t \text { times }}, u_{t+1}, \ldots)<w(\underbrace{b, \ldots, b}_{t \text { times }}, v_{t+1}, \ldots)
$$

for all $\mathbf{u}, \mathbf{v} \in \mathbf{X}$.

In particular, there exists $\tau \in \mathbb{N}$ such that,

$$
w(\underbrace{a, \ldots, a}_{\tau \text { times }}, 1_{\text {con }})<w(\underbrace{b, \ldots, b}_{\tau \text { times }}, 0_{\text {con }}) .
$$

So in the comparison of constant streams, only what happens before a finite $\tau$ matters. Note that $\tau$ depends on $a$ and $b$.

Proof Assume Continuity, Strong Pareto, and Stationarity, and let $w$ have the property that $w\left(c_{\mathrm{con}}\right)=c$ for all $c \in[0,1]$. Note that the aggregator function $g$ satisfies $(\mathrm{G} .1)-$ (G.4). Define the sequence $\left\{a^{t}\right\}$ by: 


$$
\begin{aligned}
a^{1}= & g(a, 1)=w\left(a, 1_{\text {con }}\right) \\
a^{2}= & g\left(a, a^{1}\right)=w\left(a, a, 1_{\text {con }}\right) \\
& \ldots \\
a^{t}= & g\left(a, a^{t-1}\right)=w(\underbrace{a, \ldots, a}_{t \text { times }}, 1_{\text {con }}) .
\end{aligned}
$$

For all $t, a^{t} \in(a, 1)$. Furthermore, $\left\{a^{t}\right\}$ is decreasing and bounded below by $a$. Hence $\left\{a^{t}\right\}$ is a converging sequence: $a^{\infty}:=\lim _{t \rightarrow \infty} a^{t} \in[a, 1)$. By continuity of $g$, $a^{\infty}=g\left(a, a^{\infty}\right)$. If $a^{\infty}>a$, then

$$
a^{\infty}=g\left(a, a^{\infty}\right)<g\left(a^{\infty}, a^{\infty}\right)=a^{\infty},
$$

which is a contradiction. Therefore: $a^{\infty}=a$.

Define likewise the sequence $\left\{b^{t}\right\}$ by $b^{t}=g\left(b, b^{t-1}\right)$ for all $t \in \mathbb{N}$ and $b^{0}=0$. The same argument implies that $b^{\infty}=b$.

Hence, with $a<b$, we obtain

$$
\lim _{t \rightarrow \infty} w(\underbrace{a, \ldots, a}_{t \text { times }}, 1_{\text {con }})=a<b=\lim _{t \rightarrow \infty} w(\underbrace{b, \ldots, b}_{t \text { times }}, 0_{\text {con }})
$$

Hence, by Strong Pareto there exists $\tau \in \mathbb{N}$ such that

$$
\begin{aligned}
& w(\underbrace{a, \ldots, a}_{t \text { times }}, u_{t+1}, \ldots) \leq w(\underbrace{a, \ldots, a}_{\tau \text { times }}, 1_{\text {con }}) \\
&<w(\underbrace{b, \ldots, b}_{\tau \text { times }}, 0_{\text {con }}) \leq w(\underbrace{b, \ldots, b}_{t \text { times }}, v_{t+1}, \ldots)
\end{aligned}
$$

for all $t \geq \tau$ and $\mathbf{u}, \mathbf{v} \in \mathbf{X}$, thereby establishing the result.

The following result generalizes Observation 1 by showing that only what happens before a finite $\tau$ matters even in the comparisons of converging streams that need not be constant.

Proposition 1 There exists no monotone SwF $w$ that satisfies Continuity, Strong Pareto, Stationarity, and Non-dictatorship of the present (on the set of converging streams).

In the remainder of this section we demonstrate the tightness of this result by showing how the removal of any one of these axioms leads to possibility.

Removing Non-dictatorship of the present (on the set of converging streams) The TDU SWF $w_{\beta}^{T}$, as defined by (T), is monotone and satisfies Continuity, Strong Pareto, and Stationarity. ${ }^{3}$

\footnotetext{
3 It is an open question whether Continuity, Strong Pareto, and Stationarity can be combined with Nondictatorship of the present when the latter axiom is weakened to allow for the use of non-convergent stream $\mathbf{x}$ and $\mathbf{y}$ satisfying that not only what happens before a finite time matters for the ranking of these alternatives. We cannot establish Proposition 1 with this weaker axiom but, on the other hand, we have no counterexample showing that these axioms are compatible.
} 
Weakening Strong Pareto to Restricted dominance The Sustainable discounted utilitarian SWF $w_{\beta}^{S}: \mathbf{X} \rightarrow[0,1]$ discounts the future by $\beta \in(0,1)$ if and only if the future is better than the present. It is defined by $w_{\beta}^{S}(\mathbf{x})=\lim _{\tau \rightarrow \infty} u^{-1}(z(1, \tau))$, where, for each $\tau \in \mathbb{N}, z(1, \tau)$ is constructed as follows:

$$
\begin{aligned}
z(\tau, \tau)= & u\left(w_{\beta}^{T}\left(x_{\tau}, x_{\tau+1}, \ldots\right)\right) \\
z(\tau-1, \tau)= & \min \left\{(1-\beta) u\left(x_{\tau-1}\right)+\beta z(\tau, \tau), z(\tau, \tau)\right\} \\
& \ldots \\
z(1, \tau)= & \min \left\{(1-\beta) u\left(x_{1}\right)+\beta z(2, \tau), z(2, \tau)\right\} .
\end{aligned}
$$

The analysis of Asheim and Mitra (2010) implies that $w_{\beta}^{S}$ is monotone and satisfies Continuity, Restricted dominance, Stationarity, and Non-dictatorship of the present (on the set of converging streams).

Weakening Stationarity to Separable future The Chichilnisky SWF $w^{C}: \mathbf{X}^{c} \rightarrow$ $[0,1]$, being in the class considered in Chichilnisky's (1996) Theorems 1 and 2, evaluates converging streams according to a convex combination of TDU welfare and the limit of wellbeing:

$$
w^{C}(\mathbf{x})=(1-\gamma) w_{\beta}^{T}(\mathbf{x})+\gamma \lim _{t \rightarrow \infty} x_{t},
$$

where $\gamma \in(0,1)$. The analysis of Chichilnisky (1996) implies that $w^{C}$ is monotone and satisfies Continuity, Strong Pareto, Non-dictatorship of the present (on the set of converging streams) as well as the following separability axiom: ${ }^{4}$

Separable future. For all $\mathbf{x}, \mathbf{y}, \mathbf{x}^{\prime}, \mathbf{y}^{\prime} \in \mathbf{X}$ such that (i) $x_{t}=x_{t}^{\prime}$ and $y_{t}=y_{t}^{\prime}$ for all $t \in \mathbb{N} \backslash\{1\}$ and (ii) $x_{1}=y_{1}$ and $x_{1}^{\prime}=y_{1}^{\prime}, w(\mathbf{x}) \geq w(\mathbf{y})$ if and only if $w\left(\mathbf{x}^{\prime}\right) \geq w\left(\mathbf{y}^{\prime}\right)$.

This is Koopmans' (1960) Postulate 3b. It is implied by Stationarity, since by the latter axiom, $w(\mathbf{x}) \geq w(\mathbf{y})$ if and only if $w\left(\mathbf{x}^{\prime \prime}\right) \geq w\left(\mathbf{y}^{\prime \prime}\right)$, and $w\left(\mathbf{x}^{\prime}\right) \geq w\left(\mathbf{y}^{\prime}\right)$ if and only if $w\left(\mathbf{x}^{\prime \prime}\right) \geq w\left(\mathbf{y}^{\prime \prime}\right)$, where $x_{t}^{\prime \prime}=x_{t+1}=x_{t+1}^{\prime}$ and $y_{t}^{\prime \prime}=y_{t+1}=y_{t+1}^{\prime}$ for all $t \in \mathbb{N}$.

One gets another perspective on the non-stationarity of the Chichilnisky SWF $w^{C}$ by noting that, on the set $\mathbf{X}^{c}$ of converging streams, the limit of wellbeing equals the limit of TDU welfare as the discount factor goes to 1 . Hence, the SWF given by (C) can be rewritten as:

$$
w^{C}(\mathbf{x})=(1-\gamma) w_{\beta}^{T}(\mathbf{x})+\gamma \lim _{\delta \rightarrow 1} w_{\delta}^{T}(\mathbf{x})
$$

This means that $w^{C}$ might be looked at as a Pareto-efficient and non-dictatorial aggregation of heterogeneous preferences, with $(1-\gamma)$ weight on TDU and $\gamma$ weight on undiscounted utilitarianism. Thus, as shown by Jackson and Yariv (2015, Proposition 1) and interpreted by Millner and Heal (2018), $w^{C}$ is non-stationary.

\footnotetext{
${ }^{4}$ We verify in "Appendix B" that the Chichilnisky SwF $w^{C}$ does not satisfy Stationarity.
} 
Weakening Continuity to Limited discontinuity We provide two examples of representable and monotone SWFs that satisfy Limited discontinuity, Strong Pareto, Stationarity and Non-dictatorship of the present (on the set of converging streams).

Example 1 the SWF $w^{1}: \mathbf{X} \rightarrow\left[0, \frac{3}{2}\right]$ be given by:

$$
w^{1}(\mathbf{x})= \begin{cases}\frac{1}{2}+(1-\beta) \sum_{t=1}^{\infty} \beta^{t-1} x_{t} & \text { if, for all } t \in \mathbb{N}, x_{t} \geq \frac{1}{2}, \\ (1-\beta) \sum_{t=1}^{\infty} \beta^{t-1} x_{t} & \text { if there is } t \in \mathbb{N} \text { such that } x_{t}<\frac{1}{2}\end{cases}
$$

where $\beta \in(0,1)$. The SWF $w^{1}$ does not satisfy Continuity. To see this, note that $w^{1}\left(a, b_{\text {con }}\right)=\frac{1}{2}$ if $a=\frac{1}{2}-\frac{1}{2} \beta$ and $b=1-\frac{1}{2} \beta$. However, $w^{1}\left(c_{\text {con }}\right)=c<\frac{1}{2}=$ $w^{1}\left(a, b_{\text {con }}\right)$ if $c \in\left[0, \frac{1}{2}\right)$ while $w^{1}\left(\frac{1}{2}{ }_{\text {con }}\right)=\frac{1}{2}+\frac{1}{2}>\frac{1}{2}=w^{1}\left(a, b_{\text {con }}\right)$. Hence, there is no positive monotonic transformation $v$ of $w^{1}$ with the property that $\lim _{c \uparrow \frac{1}{2}} v\left(c_{\text {con }}\right)=$ $v\left(\frac{1}{2}_{\text {con }}\right)$, since $w^{1}(\mathbf{X})=\left[0, \frac{3}{2}\right]$ and $f_{w^{1}}([0,1])=\left[0, \frac{1}{2}\right) \cup\left[1, \frac{3}{2}\right]$.

Example 2 Define the following subsets of $\mathbf{X}: \mathbf{N}=\left\{\mathbf{x} \in \mathbf{X}: \sum_{t=1}^{\infty} x_{t}<\infty\right\}$ and $\mathbf{I}=\mathbf{X} \backslash \mathbf{N}$. Let the SWF $w^{2}: \mathbf{X} \rightarrow[0,2]$ be given by:

$$
w^{2}(\mathbf{x})= \begin{cases}1+(1-\beta) \sum_{t=1}^{\infty} \beta^{t-1} x_{t} & \text { if } \mathbf{x} \in \mathbf{I} \\ \frac{\sum_{t=1}^{\infty} x_{t}}{1+\sum_{t=1}^{\infty} x_{t}} & \text { if } \mathbf{x} \in \mathbf{N}\end{cases}
$$

where $\beta \in(0,1)$. The SWF $w^{2}$ does not satisfy Continuity. To see this, note that $w^{2}\left(b_{\text {con }}\right)>1>w^{2}\left(1,0_{\text {con }}\right)=\frac{1}{2}>w^{2}\left(0_{\text {con }}\right)=0$ if $b \in(0,1]$. Hence, there is no positive monotonic transformation $v$ of $w^{2}$ with the property that $\lim _{b \downarrow} v\left(b_{\text {con }}\right)=$ $v\left(0_{\text {con }}\right)$.

The SWF $w^{2}$ of Example 2 represents time-discounted utilitarianism on the set $\mathbf{I}$ of streams with unbounded sum of wellbeing. If subsistence requires wellbeing bounded away from zero, then $\mathbf{I}$ is the relevant set of streams in the context of sustainability. The discontinuity between streams in $\mathbf{I}$ and streams in $\mathbf{N}$ is an artifact to ensure that the SWF $w^{2}$ satisfies Non-dictatorship of the present (on the set of converging streams). A similar comment can be made in the case of Example 1 with $\frac{1}{2}$ as subsistence level. Chichilnisky (1996) defines 'sustainable preference' as a SWF that satisfies Strong Pareto and Non-dictatorship of the present (noting that her axiom Non-dictatorship of the future follows from Strong Pareto). Examples 1 and 2 show that there exist 'sustainable preferences' that are stationary. However, they illustrate also that axioms discussed in the literature actually fail to put adequate restrictions on SWFs, and the commonly accepted characterization results obtained from these axioms are driven by the imposition of Continuity. In particular, a 'sustainable preference' without the additional imposition of Continuity might not have commendable properties. Thus, the dilemma posed by a choice between Stationarity and intergenerational equity remains. 


\section{Stationarity leads to impatience}

In this section we show how combinations of axioms including Stationarity imply impatience. We start by the observation that $a<b$ implies $w\left(a, b, b_{c o n}\right)<$ $w\left(b, a, b_{c o n}\right)$ if $w$ is a monotone SWF on $\mathbf{X}$ satisfying Continuity, Strong Pareto, and Stationarity. To see this, suppose instead that $w\left(a, b_{\text {con }}\right) \geq w\left(b, a, b_{\text {con }}\right)$. Then, by Stationarity, $w\left(b, a, b_{\mathrm{con}}\right) \geq w\left(b, b, a, b_{\mathrm{con}}\right) \geq w\left(b, b, b, a, b_{\mathrm{con}}\right) \geq \ldots$ and, by Strong Pareto and the Proof of Observation 1,

$$
w(\underbrace{b, \ldots, b}_{t \text { times }}, a, b_{\text {con }}) \geq w(\underbrace{b, \ldots, b}_{t \text { times }}, 0_{\text {con }}) \rightarrow w\left(b_{\text {con }}\right)>w\left(a, b_{\text {con }}\right) \text { as } t \rightarrow \infty .
$$

This contradicts $w\left(a, b_{\text {con }}\right) \geq w\left(b, a, b_{\text {con }}\right)$ and shows how the axioms of Continuity, Strong Pareto, and Stationarity are in conflict with equal treatment of generations in the sense of Finite anonymity. The result that $a<b$ implies $w\left(a, b, b_{\text {con }}\right)<$ $w\left(b, a, b_{\text {con }}\right)$ actually follows from part (a) of Koopmans's (1960) Theorem 1 , and it meets his condition (40) of impatience.

However, as shown by Basu and Mitra (2003), equal treatment of generations is ruled out under Strong Pareto even without imposing Continuity and Stationarity, as no SWF can satisfy both Strong Pareto and Finite anonymity. Hence, we must impose a weaker sensitivity axiom than Strong Pareto to show that Stationarity has a key role in undermining equal treatment.

The following main result of this section shows that Finite anonymity is contradicted by Stationarity even if Continuity is weakened to Limited discontinuity and Strong Pareto is weakened to Restricted dominance. The significance of this result is that Limited discontinuity and Restricted dominance alone do not contradict Finite anonymity. Hence, under these two axioms it is the requirement of Stationarity that causes the conflict with equal treatment as a condition for intergenerational equity, as we observe below in our discussion of the tightness of the result.

Proposition 2 There exists no monotone SWF $w$ that satisfies Limited discontinuity, Restricted dominance, Stationarity, and Finite anonymity.

Removing Finite anonymity The TDU SWF $w_{\beta}^{T}$, as defined by (T), is monotone and satisfies Limited discontinuity, Restricted dominance, and Stationarity.

Weakening Stationarity to Weak stationarity The Rank-discounted utilitarian (RDU) SWF $w_{\beta}^{R}: \mathbf{X} \rightarrow[0,1]$ defined by:

$$
w_{\beta}^{R}(\mathbf{x})=\inf _{\pi \in \Pi} w_{\beta}^{T}\left(\mathbf{x}_{\pi}\right)
$$

where $\Pi$ denote the set of all (also infinite) permutations of $\mathbb{N}$. The definition given by (R) is equivalent to Zuber and Asheim's (2012) Definition 2, implying that $w_{\beta}^{R}$ discounts according to rank, not according to time as $w_{\beta}^{T}$ does. Their analysis shows 
that $w_{\beta}^{R}$ is a monotone SWF that satisfies Limited discontinuity, Restricted dominance, Finite anonymity as well as the following weakening of Stationarity: ${ }^{5}$

Weak stationarity There exists $a \in[0,1]$ such that, for all $\mathbf{x}, \mathbf{y} \in \mathbf{X}$ with $x_{1}=y_{1}=a$, $w(\mathbf{x}) \geq w(\mathbf{y})$ if and only if $w\left(\mathbf{x}^{\prime}\right) \geq w\left(\mathbf{y}^{\prime}\right)$, where $x_{t}^{\prime}=x_{t+1}$ and $y_{t}^{\prime}=y_{t+1}$ for all $t \in \mathbb{N}$.

This is satisfied by $w_{\beta}^{R}$ by choosing $a=0$ (or $a=1$, given our assumption that the set of possible wellbeings equals the unit interval). The axiom is Koopmans' (1960) Postulate 4, and it follows from Stationarity since, by the latter axiom, the equivalence of Weak stationarity holds for all $a \in[0,1]$. Conversely, in conjunction with Separable future it implies Stationarity. Also, the maximin SWF $w^{M}$, defined by $w^{M}(\mathbf{x})=\inf _{t \in \mathbb{N}} x_{t}$, is monotone and satisfies Limited discontinuity, Restricted dominance, and Finite anonymity, as well as Weak stationarity by choosing $a=1$.

Weakening Restricted dominance We provide two examples of monotone SWFs that satisfy Limited discontinuity, Stationarity, and Finite anonymity. The first one, which is a variant of Example 2, retains sensitivity only for summable streams, while the second one has only asymptotic sensitivity.

Example 3 As in Example 2, let $\mathbf{N}=\left\{\mathbf{x} \in \mathbf{X}: \sum_{t=1}^{\infty} x_{t}<\infty\right\}$ and $\mathbf{I}=\mathbf{X} \backslash \mathbf{N}$. Let the $\mathrm{SWF} w^{3}: \mathbf{X} \rightarrow[0,1]$ be given by:

$$
w^{3}(\mathbf{x})= \begin{cases}1 & \text { if } \mathbf{x} \in \mathbf{I}, \\ \frac{\sum_{t=1}^{\infty} x_{t}}{1+\sum_{t=1}^{\infty} x_{t}} & \text { if } \mathbf{x} \in \mathbf{N}\end{cases}
$$

The SWF $w^{3}$ does not satisfy Restricted dominance as $w^{3}\left(a, b_{\text {con }}\right)=1=w^{3}\left(b_{\text {con }}\right)$ if $a<b$, while satisfying Strong Pareto on the set of summable streams.

Example 4 Let the SWF $w^{4}: \mathbf{X} \rightarrow[0,1]$ be given by:

$$
w^{4}(\mathbf{x})=\alpha \liminf \inf _{t \rightarrow \infty} x_{t}+(1-\alpha) \lim \sup _{t \rightarrow \infty} x_{t},
$$

where $\alpha \in(0,1)$. Even though $w^{4}$ does not satisfy Restricted dominance, $w^{4}$ has the property that $w^{4}\left(c_{\text {con }}\right)=c$ for all $c \in[0,1]$. In particular, the SWF satisfies a "uniform" Pareto principle in the sense that for all $\mathbf{x}, \mathbf{y} \in \mathbf{X}$ and $\varepsilon>0$, if $x_{t} \geq y_{t}+\varepsilon$ for all $t \in \mathbb{N}$, then $w^{4}(\mathbf{x})>w^{4}(\mathbf{y})$. For more on the axiomatic basis for related SWFs, see Chambers (2009) and Sakai (2016).

These two examples illustrate that a SWF that is stationary and treats generations equally cannot have both sensitivity for the present generation and asymptotic sensitivity. In fact, Restricted dominance is a minimalist way of capturing both types of sensitivity. And as soon as one imposes Restricted dominance (in combination with Limited discontinuity), we know that Stationarity lead to impatience, so equal treatment as captured by Finite anonymity is destroyed.

\footnotetext{
5 We verify in "Appendix B" that the RDU SWF $w_{\beta}^{R}$ does not satisfy Stationarity.
} 
Removing Limited discontinuity We end this section by noting that there is another route out of the dilemma discussed here, namely to drop the minimal continuity requirement imposed by Limited discontinuity together with the assumption that the SWR is representable. Undiscounted utilitarianism and leximin as defined in the setting of infinite wellbeing streams by Basu and Mitra (2007) and Bossert et al. (2007), respectively, are reflexive, transitive, and monotone SWRs that satisfy Finite anonymity as well as Strong Pareto and Stationarity, and thus all the remaining axioms of Proposition 2, as Strong Pareto implies Restricted dominance. However, even if we impose completeness (necessarily by the use of non-constructive mathematics, see Zame 2007; Lauwers 2010), they are not representable, precisely because there is no limit to the discontinuity of such SWRs on the diagonal of $\mathbf{X}$, i.e. on the subset of constant streams.

We conjecture that no monotone SWF $w$ satisfying Restricted dominance, Stationarity, and Finite anonymity can negate Limited discontinuity by having the property that the set $D\left(f_{w}\right)$ is dense in $[0,1]$. This means that the requirements of representability and monotonicity combined with Restricted dominance, Stationarity, and Finite anonymity is sufficient to obtain non-existence, so that the axiom of Limited discontinuity is not needed for Proposition 2. We discuss this conjecture in the remark at the end of "Appendix A".

\section{Time consistency or time invariance?}

The consequences of non-stationary SWFs can be analyzed in a setting where social rankings are made at any time $\tau \in \mathbb{N}$. Hence, for all $\mathbf{x} \in \mathbf{X}$ and any $\tau \in \mathbb{N}$, write ${ }_{\tau} \mathbf{x}=\left(x_{\tau}, x_{\tau+1}, \ldots\right)$, and let $w_{\tau}$ denote a SWF defined for streams starting at time $\tau$. In this setting one can impose the axioms of Stationarity, Time invariance and Time consistency at any $\tau \in \mathbb{N}$ :

Stationarity For any $\tau \in \mathbb{N}$ and all $\mathbf{x}, \mathbf{y} \in \mathbf{X}$ with $x_{\tau}=y_{\tau}, w_{\tau}\left({ }_{\tau} \mathbf{x}\right) \geq w_{\tau}\left({ }_{\tau} \mathbf{y}\right)$ if and only if $w_{\tau}\left({ }_{\tau} \mathbf{x}^{\prime}\right) \geq w_{\tau}\left({ }_{\tau} \mathbf{y}^{\prime}\right)$, where $x_{t}^{\prime}=x_{t+1}$ and $y_{t}^{\prime}=y_{t+1}$ for all $t \geq \tau$.

Time invariance For any $\tau \in \mathbb{N}$ and all $\mathbf{x}, \mathbf{y} \in \mathbf{X}, w_{\tau}\left({ }_{\tau} \mathbf{x}\right) \geq w_{\tau}\left({ }_{\tau} \mathbf{y}\right)$ if and only if $w_{\tau+1}\left({ }_{\tau+1} \mathbf{x}^{\prime}\right) \geq w_{\tau+1}\left({ }_{\tau+1} \mathbf{y}^{\prime}\right)$, where $x_{t+1}^{\prime}=x_{t}$ and $y_{t+1}^{\prime}=y_{t}$ for all $t \geq \tau$.

Time consistency For any $\tau \in \mathbb{N}$ and all $\mathbf{x}, \mathbf{y} \in \mathbf{X}$ with $x_{\tau}=y_{\tau}, w_{\tau}\left({ }_{\tau} \mathbf{x}\right) \geq w_{\tau}\left({ }_{\tau} \mathbf{y}\right)$ if and only if $w_{\tau+1}\left({ }_{\tau+1} \mathbf{x}\right) \geq w_{\tau+1}\left({ }_{\tau+1} \mathbf{y}\right)$.

The following result is well-known, see, for example, (Halevy, 2015, Proposition 4).

Proposition 3 Any two of the three axioms: Stationarity, Time invariance, and Time consistency, imply the third.

Hence, if Stationarity does not hold, then we must choose between Time consistency and Time invariance; we cannot satisfy both.

One route is to insist on Time consistency and allow SWFs to be time variant. In the case of the Chichilnisky SWF $w^{C}$ this means that the weight on the TDU part of $w^{C}$ vanishes as time goes to infinity, so that asymptotically only the limit of wellbeing matters. In the case of the RDU SWF $w_{\beta}^{R}$, insistence of Time consistency and relaxation of Time invariance makes $w_{\beta}^{R}$ history dependent, as past wellbeing levels must be 
allowed to enter into the ranking of future wellbeing levels. In the case of the maximin SWF $w^{M}$ the consequence of this route is particularly stark, since all future streams become equally good as long as the minimal wellbeing in the past is smaller than the infimum of future wellbeing.

An alternative route is to insist on Time invariance, which corresponds to consequentialist social decision-making in terms of the future properties of the stream. In this setting we can investigate the consequences of sophisticated planning as a rational manner to tackle the resulting time inconsistency. In the remainder of this section we do so by applying the Chichilnisky and RDU SWFs in the context of the Ramsey model (Ramsey 1928). In this model, at each $t \in \mathbb{N}$ net production, $f\left(k_{t-1}\right)$, depends on the stock of capital, $k_{t-1}$, and is split between wellbeing, $x_{t}$, and net accumulation of capital, $k_{t}-k_{t-1}$ :

$$
x_{t}+\left(k_{t}-k_{t-1}\right)=f\left(k_{t-1}\right) \text {, }
$$

with $k_{0}=k$ as initial condition. Capital is assumed to be non-negative, and the net production function $f: \mathbb{R}_{+} \rightarrow \mathbb{R}_{+}$is assumed to be increasing, strictly concave and continuously differentiable, with $f(0)=0, \lim _{k \rightarrow 0} f^{\prime}(k)=\infty$ and $\lim _{k \rightarrow \infty} f^{\prime}(k)=$ 0 . Assume also that the utility function $u: \mathbb{R}_{+} \rightarrow \mathbb{R}$, in addition to being an increasing and continuous function, is strictly concave, continuously differentiable, and satisfies $\lim _{x \rightarrow 0} u^{\prime}(x)=\infty$. Note that we, for the analysis of this section, allow wellbeing to take on values in the set all non-negative real numbers, $\mathbb{R}_{+}$, so the restriction to $[0,1]$ is relaxed and the set of possible wellbeing streams $\mathbf{X}$ equals $\mathbb{R}_{+}^{|\mathbb{N}|}$.

A Markov strategy $\sigma$ maps from the capital stock to a feasible flow of wellbeing:

$$
\sigma: k \in \mathbb{R}_{+} \mapsto \sigma(k) \in[0, k+f(k)]
$$

For given initial condition $k$, a Markov strategy $\sigma$ implements a capital stream:

$$
\begin{aligned}
\mathbf{k}(\sigma, k) & =\left(k_{0}, k_{1}, \ldots, k_{t}, \ldots\right) \\
\text { where } k_{0} & =k \text { and, for all } t \in \mathbb{N}, k_{t}=k_{t-1}+f\left(k_{t-1}\right)-\sigma\left(k_{t-1}\right),
\end{aligned}
$$

and a wellbeing stream:

$$
\mathbf{x}(\sigma, k)=\left(x_{1}, x_{2}, \ldots, x_{t}, \ldots\right) \text { where for all } t \in \mathbb{N}, x_{t}=\sigma\left(k_{t-1}\right) .
$$

A Markov strategy $\sigma$ is a Markov-perfect equilibrium under a SWF $w: \mathbf{X} \rightarrow \mathbb{R}$ if:

For all $k \in \mathbb{R}_{+}, w(\mathbf{x}(\sigma, k))=\max _{x_{1} \in[0, k+f(k)]} w\left(x_{1}, x_{2}, \ldots, x_{t}, \ldots\right)$,

with $\mathbf{x}^{\prime}=\mathbf{x}\left(\sigma, k+f(k)-x_{1}\right)$ where $x_{t}^{\prime}=x_{t+1}$ for all $t \in \mathbb{N}$. Using the concept of a Markov-perfect equilibrium is a common way of modeling sophisticated planning under time inconsistency. ${ }^{6}$

\footnotetext{
6 Examples of Markov-perfect equilibrium analysis in the context of intertemporal resource allocation include Dockner and Wagener (2014) in continuous time and Sorger (1998) and Mitra and Sorger (2014) in discrete time.
} 
It follows from Beals and Koopmans (1969) that there is a unique TDU optimum, with both capital and consumption streams being strictly monotone, with capital converging to $k_{\infty}(\beta)$, and with wellbeing converging to $f\left(k_{\infty}(\beta)\right)$, where $k_{\infty}(\beta)$ is defined by $\beta\left(1+f^{\prime}\left(k_{\infty}(\beta)\right)=1\right.$. Since the capital stream is strictly monotone, there exists a TDU Markov strategy, $\sigma^{T}$, which implements the TDU optimum. Clearly, $\sigma^{T}$ is a Markov-perfect equilibrium under the TDU SWF $w_{\beta}^{T}$, because otherwise it would have been possible to improve upon the TDU optimum.

Under the Chichilnisky SWF $w^{C}$ there is no optimal stream in the Ramsey model. The reason is that it is profitable to delay the concern for the infinite future, while infinite delay is worse. Furthermore, it is straightforward to argue that $\sigma^{T}$ is a Markovperfect equilibrium under $w^{C}$. The reason is that there is no possibility for any one generation to influence the infinite future, since under $\sigma^{T}$ wellbeing converges to $f\left(k_{\infty}(\beta)\right)$ independently of the initial capital stock. Hence, it is a best reply for each generation to maximize the TDU SWF $w_{\beta}^{T}$, which is what is achieved by following the TDU Markov strategy $\sigma^{T}$.

There are other and better non-Markovian equilibria under $w^{C}$. To see this, let $\sigma_{\left[k^{*}, \infty\right)}^{T}$ be the Markov strategy that implements the TDU optimum given that the stock of capital is constrained to remain at least $k^{*}$, where $k^{*}>k_{\infty}(\beta)$. Let a non-Markovian strategy $\left(\Sigma_{1}, \Sigma_{2}, \ldots, \Sigma_{t}, \ldots\right)$ be defined by, for all $t \in \mathbb{N}$ :

$$
\Sigma_{t}\left(k_{0}, k_{1}, \ldots, k_{t-1}\right)= \begin{cases}\sigma^{T}\left(k_{t-1}\right) & \text { if } \exists \tau \in\{0, \ldots, t-1\} \text { such that } k_{\tau}<k^{*} \\ \sigma_{\left[k^{*}, \infty\right)}^{T}\left(k_{t-1}\right), & \text { otherwise. }\end{cases}
$$

Hence, if the initial capital stock $k$ is at least as large as $k^{*}>k_{\infty}(\beta)$, following $\left(\Sigma_{1}, \Sigma_{2}, \ldots, \Sigma_{t}, \ldots\right)$ implies that $k_{t}$ converges to $k^{*}$. For an interval of $k^{*}$ values exceeding $k_{\infty}(\beta)$ this is an equilibrium strategy, as the loss in terms of TDU welfare is more than compensated for by a higher limit of wellbeing. Asheim and Ekeland (2016) show how such equilibria can be Markovian in continuous time.

Under the RDU SWF $w_{\beta}^{R}$ there exists a unique optimum for any $k \in \mathbb{R}_{+}$(Zuber and Asheim 2012, Proposition 10). This optimum can be implemented by the following Markov strategy whereby the TDU optimum is implemented with a small initial capital stock (corresponding to a high initial capital productivity) and the maximin stream is implemented with a large initial capital stock (corresponding to a low initial capital productivity):

$$
\sigma^{R}(k)= \begin{cases}\sigma^{T}(k) & \text { if } k<k_{\infty}(\beta), \\ f(k), & \text { if } k \geq k_{\infty}(\beta) .\end{cases}
$$

Furthermore, $\sigma^{R}$ is a Markov-perfect equilibrium under the RDU SWF $w_{\beta}^{R}$. Hence, for any $k \in \mathbb{R}_{+}$, the RDU optimum is time consistent.

However, there are also other and worse Markov-perfect equilibria under $w_{\beta}^{R}$. The following is one example of a Markov-perfect equilibrium where capital is depleted asymptotically, so that the limit of wellbeing as time goes to infinity equals zero:

$$
\sigma(k)=f(k)+\delta k \text { for all } k, \delta \in(0,1]
$$


This is a Markov-perfect equilibrium as any one generation cannot prevent that the limit of wellbeing as time goes to infinity equals zero, so that all present wellbeing choices are equally bad. The existence of this Markov-perfect equilibrium is caused by the RDU SWF $w_{\beta}^{R}$ not being continuous at infinity. The existence of such unattractive Markov-perfect equilibria calls for refinements that pick out the unique RDU optimum, as it is the obvious consequence of sophisticated planning to choose the best stream that will actually be followed under $w_{\beta}^{R}$. Revision-proof equilibrium (Asheim 1997) is a refinement that works in this case.

Our application of the Chichilnisky and RDU SWFs to the Ramsey model shows how non-stationary SWFs of intergenerational equity can be applied in a growth model, even if one insists on Time invariance and, thus, is forced to handle time inconsistency. However, there is a multiplicity of equilibria, suggesting that the principles for equilibrium selection must be carefully considered.

\section{Concluding remarks}

In this paper we have shown how conditions for intergenerational equity may necessitate the non-stationarity of normative criteria. One might claim that, while non-stationarity appears to be pervasive in individual decision-making, it has no place when formulating normative criteria for rational social decision-making. If so, our results would indicate that it might be difficult to base rational social decision-making on principles for intergenerational distributive justice.

We disagree with this view for two reasons. Firstly, non-stationarity is not irrational; the question of rationality in this context relates to whether the decision-maker (be it an individual person or a social planner) is conscious of his non-stationarity and, if so, how this feature of preferences is handled. A consequentialist rational social planner with non-stationary and time invariant, thus time inconsistent, preferences must be aware that optimal plans might not be followed and instead plan in a "sophisticated" way by choosing the best plans that will actually be followed (see Pollak 1968; Blackorby et al. 1973, for early references).

Secondly, several normative criteria of intergenerational equity are in fact nonstationary. Examples that we have discussed in this paper are the Chichilnisky and rank-discounted utilitarian social welfare functions, in addition to the more wellknown maximin criterion. If non-stationarity is unavoidable for any "attractive" criterion satisfying equity axioms like anonymity or no dictatorship of the present under reasonable sensitivity, coherency, richness and robustness conditions, then we must face the intriguing problem of how to apply these criteria for normative social choice. We have illustrated that such application is indeed possible, even though it leads to interesting game-theoretic problems related to equilibrium selection.

Acknowledgements Open Access funding provided by University of Oslo (incl Oslo University Hospital) 


\section{Compliance with ethical standards}

Funding No funding was received.

Conflict of interest Asheim and Banerjee declare no conflicts of interest and no competing interests. Mitra is deceased; Asheim and Banerjee do not know of any conflicts of interest or any competing interests in his case.

Availability of data and material (data transparency) Not applicable.

Code availability (software application or custom code) Not applicable.

Open Access This article is licensed under a Creative Commons Attribution 4.0 International License, which permits use, sharing, adaptation, distribution and reproduction in any medium or format, as long as you give appropriate credit to the original author(s) and the source, provide a link to the Creative Commons licence, and indicate if changes were made. The images or other third party material in this article are included in the article's Creative Commons licence, unless indicated otherwise in a credit line to the material. If material is not included in the article's Creative Commons licence and your intended use is not permitted by statutory regulation or exceeds the permitted use, you will need to obtain permission directly from the copyright holder. To view a copy of this licence, visit http://creativecommons.org/licenses/by/4.0/.

\section{Appendix A: Proofs of Propositions 1 and 2}

In Proposition 1 we need to show that there is no monotone SWF satisfying the axioms of Continuity, Strong Pareto, Stationarity and Non-dictatorship of the present (on the set of converging streams). The strategy of the proof is to demonstrate that any monotone SWF $w$ satisfying Continuity, Strong Pareto, Stationarity must violate Non-dictatorship of the present (on the set of converging streams). To elaborate, the following will be shown, where $w$ has the property that $w\left(c_{\text {con }}\right)=c$ for all $c \in[0,1]$ and where the associated aggregator function $g$ (as introduced in Sect. 2) satisfies G.1-G.4: For any $\mathbf{x}, \mathbf{y} \in \mathbf{X}^{c}$ satisfying $w(\mathbf{x})<w(\mathbf{y})$, there is some $\tau \in \mathbb{N}$ such that we have $w\left(x_{1}, \cdots, x_{t}, 1_{\text {con }}\right)<w\left(y_{1}, \cdots, y_{t}, 0_{\text {con }}\right)$ for all $t \geq \tau$, in violation of Nondictatorship of the present (on the set of converging streams).

For the statement of next lemma, define for all $\mathbf{x} \in \mathbf{X}$ the sequences $\left\{\ell_{t}(\mathbf{x})\right\}$ and $\left\{h_{t}(\mathbf{x})\right\}$ as follows:

For all $t \in \mathbb{N}, \ell_{t}(\mathbf{x})=w\left(x_{1}, \ldots, x_{t}, 0_{\text {con }}\right)$ and $h_{t}(\mathbf{x})=w\left(x_{1}, \ldots, x_{t}, 1_{\text {con }}\right)$.

Clearly, $\left\{\ell_{t}(\mathbf{x})\right\}$ is a nondecreasing sequence, bounded above by $w(\mathbf{x})$ by Strong Pareto. So it converges to a limit; denote this limit by $L(\mathbf{x})$. Similarly, using Strong Pareto, the sequence $\left\{h_{t}(\mathbf{x})\right\}$ is a nonincreasing sequence, bounded below by $w(\mathbf{x})$. So it converges to a limit; denote this limit by $H(\mathbf{x})$. Hence, we have:

$$
L(\mathbf{x}) \leq w(\mathbf{x}) \leq H(\mathbf{x})
$$

Lemma 1 Assume that the monotone SWF $w$ satisfies Continuity, Strong Pareto, and Stationarity. Then $L(\mathbf{x})=w(\mathbf{x})=H(\mathbf{x})$ for all $\mathbf{x} \in \mathbf{X}^{c}$. 
Proof Assume that the monotone SWF $w$ satisfies Continuity, Strong Pareto, and Stationarity, and has the property that $w\left(c_{c o n}\right)=c$ for all $c \in[0,1]$. Let $\mathbf{x} \in \mathbf{X}^{c}$ be given, and let $a$ denote $\lim _{t \rightarrow \infty} x_{t}$. We will show that $w(\mathbf{x})=H(\mathbf{x})$. Given (3), this is established if, for every $\epsilon>0$,

there is $\tau \in \mathbb{N}$ such that $w\left(x_{1}, \ldots, x_{t}, 1_{\text {con }}\right)<w(\mathbf{x})+\epsilon$ for all $t \geq \tau$.

Let $\epsilon>0$ be given. Denote by $B_{\delta}(\mathbf{x})$ the open ball around $\mathbf{x}$ with radius $\delta>0$; that is: $B_{\delta}(\mathbf{x})=\left\{\mathbf{y} \in \mathbf{X}: \sup _{t \in \mathbb{N}}\left|y_{t}-x_{t}\right|<\delta\right\}$. Using Continuity we can find $\delta>0$ such that whenever $\mathbf{y} \in B_{\delta}(\mathbf{x})$, we have $|w(\mathbf{y})-w(\mathbf{x})| \leq \epsilon$.

Case 1: $a \in[0,1)$. Since $\lim _{t \rightarrow \infty} x_{t}=a$, there exists $\tau^{\prime} \in \mathbb{N}$ such that $\left|x_{t}-a\right|<\delta^{\prime}$ for all $t \geq \tau^{\prime}$ where, $0<\delta^{\prime}<\min \{(\delta / 3),(1-a)\}$. Define $\bar{a}=a+\delta^{\prime} \in[0,1)$ and the sequence $\left\{\bar{a}_{t}\right\}_{0}^{\infty}$ by $\bar{a}_{0}=1$ and $\bar{a}_{t+1}=g\left(\bar{a}, \bar{a}_{t}\right)$ for all $t \geq 0$. By arguments similar to the one presented in the proof of Observation 1 we can show that $\left\{\bar{a}_{t}\right\}$ is a decreasing sequence with $\bar{a}_{t}>\bar{a}$ for all $t$ and $\lim _{t \rightarrow \infty} \bar{a}_{t}=\bar{a}$. Hence, there exists $\tau^{\prime \prime} \geq 1$ such that $\bar{a}_{\tau^{\prime \prime}} \leq \bar{a}+\delta^{\prime}$. By construction, $x_{t}<a+\delta^{\prime}=\bar{a} \leq \bar{a}_{\tau^{\prime \prime}}$ and $x_{t} \geq a-\delta^{\prime} \geq \bar{a}-2 \delta^{\prime} \geq \bar{a}_{\tau^{\prime \prime}}-3 \delta^{\prime}>\bar{a}_{\tau^{\prime \prime}}-\delta$ for all $t \geq \tau^{\prime}$. Let $\tau:=\tau^{\prime}+\tau^{\prime \prime}$. Then, for all $t \geq \tau$ :

$$
\begin{aligned}
w\left(x_{1} \ldots x_{t}, 1_{\mathrm{con}}\right) & \left.\leq w\left(x_{1} \ldots x_{\tau}, 1_{\mathrm{con}}\right)<w(x_{1} \ldots x_{\tau^{\prime}}, \underbrace{\bar{a}, \ldots, \bar{a}}_{\tau^{\prime \prime} \text { times }}, 1_{\mathrm{con}})\right) \\
& =w\left(x_{1} \ldots x_{\tau^{\prime}},\left(\bar{a}_{\tau^{\prime \prime}}\right)_{\mathrm{con}}\right) \leq w(\mathbf{x})+.
\end{aligned}
$$

The inequalities in the first line of (4) follows from Strong Pareto and from noting that $x_{t}<a+\delta^{\prime}=\bar{a}$ for all $t \geq \tau^{\prime}$. The equality in the second line follows from Stationarity and noting that $w\left(\left(\bar{a}_{\tau^{\prime \prime}}\right)_{\text {con }}\right)=w\left(\bar{a}, \ldots, \bar{a}, 1_{\text {con }}\right)$ (where, $\bar{a}$ appears $\tau^{\prime \prime}$ times). The last inequality in (4) is a result of $\left(x_{1} \ldots x_{\tau}^{\prime},\left(\bar{a}_{\tau^{\prime \prime}}\right)_{\text {con }}\right) \in B_{\delta}(\mathbf{x})$ and Continuity.

Case 2: $a=1$. Since $\lim _{t \rightarrow \infty} x_{t}=1$, there exists $\tau \in \mathbb{N}$ such that $x_{t} \geq 1-\delta$ for all $t \geq \tau$. Hence, for all $t \geq \tau$ :

$$
w\left(x_{1}, \ldots, x_{\tau}, 1_{\text {con }}\right) \leq w\left(x_{1}, \ldots, x_{t}, 1_{\text {con }}\right)<w(\mathbf{x})+
$$

The first inequality being a consequence of Strong Pareto and the second inequality follows from noting that $\left(x_{1}, \ldots, x_{t}, 1_{\text {con }}\right) \in B_{\delta}(\mathbf{x})$ and Continuity.

The result that $w(\mathbf{x})=L(\mathbf{x})$ can be shown in an analogous manner.

Proof of Proposition 1 Assume that the monotone SWF $w$ satisfies Continuity, Strong Pareto, and Stationarity. Let $\mathbf{x}, \mathbf{y} \in \mathbf{X}^{c}$ satisfy $w(\mathbf{x})<w(\mathbf{y})$. Then, $\epsilon:=w(\mathbf{y})-$ $w(\mathbf{x})>0$. By Lemma 1 we can choose $\tau \in \mathbb{N}$ large enough so that for all $t \geq \tau$,

$$
w\left(x_{1} \ldots, x_{t}, 1_{\text {con }}\right)<w(\mathbf{x})+/ 2 \text { and } w\left(y_{1}, \ldots, y_{t}, 0_{\text {con }}\right)>w(\mathbf{y})-/ 2 .
$$

Thus, for all $t \geq \tau$, using (5) and the definition of $\epsilon$,

$$
\begin{aligned}
w\left(x_{1} \ldots, x_{t}, 1_{\text {con }}\right) & <w(\mathbf{x})+/ 2 \\
& =w(\mathbf{y})-+/ 2=w(\mathbf{y})-/ 2<w\left(y_{1}, \ldots, y_{t}, 0_{\text {con }}\right) .
\end{aligned}
$$


Let $\mathbf{u}, \mathbf{v}$ be arbitrary elements of $\mathbf{X}$. Then, by (6) and Strong Pareto,

$$
\begin{aligned}
& w\left(x_{1} \ldots, x_{t}, u_{t+1}, \ldots\right) \leq w\left(x_{1} \ldots, x_{t}, 1_{\mathrm{con}}\right)<w\left(y_{1}, \ldots, y_{t}, 0_{\mathrm{con}}\right) \\
& \quad \leq w\left(y_{1}, \ldots, y_{t}, v_{t+1}, \ldots\right)
\end{aligned}
$$

for all $t \geq \tau$. However, (7) contradicts Non-dictatorship of the present (on the set of converging streams).

We turn now to the Proof of Proposition 2. We state a lemma before proving the proposition.

Lemma 2 Assume that the monotone SWF $w$ satisfies Limited discontinuity and Restricted dominance. Then there is some $a, b \in[0,1]$ with $a<b$ and some $c \in[a, b)$ satisfying $w\left(a, b_{\mathrm{con}}\right)=w\left(c_{\mathrm{con}}\right)$.

Proof Assume that the monotone SWF $w$ Limited discountinity and Restricted dominance. By Limited discontinuity, $D\left(f_{w}\right)$ is not dense in $[0,1]$. Consequently, there is some (non-degenerate) open interval $\left(\mu, \mu^{\prime}\right)$ such that $f$ is continuous on all points in $\left(\mu, \mu^{\prime}\right)$. Take $a, b$ such that $\mu<a<b<\mu^{\prime}$ and observe that by the intermediate value theorem the set $f_{w}([a, b])$ is an interval. From monotonicity and Restricted dominance it follows that $f_{w}(a)=w\left(a_{\text {con }}\right) \leq w\left(a, b_{\text {con }}\right)<w\left(b_{\text {con }}\right)=f_{w}(b)$. This immediately implies that there exists $c \in[a, b)$ such that $w\left(c_{\mathrm{con}}\right)=f_{w}(c)=w\left(a, b_{\mathrm{con}}\right)$.

Proof of Proposition 2 Suppose that some monotone SWF $w$ satisfies Limited discontinuity, Restricted dominance, Stationarity, and Finite anonymity. By Lemma 2, there is some $a, b \in[0,1]$ with $a<b$ and some $c \in[a, b)$ satisfying

$$
w\left(a, b_{\text {con }}\right)=w\left(c_{\text {con }}\right)
$$

for some $w \in W$. By Stationarity and (8) we must have

$$
w\left(c, a, b_{\mathrm{con}}\right)=w\left(c_{\mathrm{con}}\right),
$$

and by Finite anonymity we obtain

$$
w\left(c, a, b_{\mathrm{con}}\right)=w\left(a, c, b_{\mathrm{con}}\right) .
$$

Equations (8)-(10) yield $w\left(a, b_{\text {con }}\right)=w\left(a, c, b_{\text {con }}\right)$. By applying Stationarity we get $w\left(b_{\mathrm{con}}\right)=w\left(c, b_{\mathrm{con}}\right)$, which along with $c<b$ contradicts Restricted dominance.

Remark 1 It is an immediate implication of Proposition 2 that any monotone SWF $w$ satisfying Restricted dominance, Stationarity, and Finite anonymity cannot satisfy Limited discontinuity. Thus, the subset $D\left(f_{w}\right)$ at which $f_{w}$ is discontinuous must be dense in $[0,1]$ for all $w \in W$. By monotonicity of $w$ the set $D\left(f_{w}\right)$ must also be a countable subset of $[0,1]$. We are unaware of whether dropping Limited discontinuity allows for the existence of a monotone SWF satisfying the remaining axioms of Proposition 2: Restricted dominance, Stationarity and Finite anonymity. We conjecture that 
there exists no such SWF even if Limited discontinuity is dropped, but we have neither a proof of impossibility nor an example of a SWF that satisfies the remaining axioms. This issue remains an open question.

To elaborate, the crux of the argument that leads to the impossibility is the existence of a stream $\left(a, b_{\text {con }}\right)$ which is indifferent to a constant profile $c_{\text {con }}$. So a natural direction to pursue is to show that a SWR for which the indifference class corresponding to every constant stream is degenerate cannot be represented by a real-valued function. However, even this severe lack of substitutability does not translate into non-representability. Indeed, Banerjee and Mitra (2018, Example 3) show that there exists a representable SWR on the unit square whose indifference classes are all singleton. This poses a challenge for the resolution of the open question. ${ }^{7}$

\section{Appendix B: The non-stationarity of $w^{C}$ and $w_{\beta}^{R}$}

The Chichilnisky SWF $w^{C}$ satisfies Separable future (as defined in Sect. 3), but not Stationarity. In fact, $w^{C}$ does not even satisfy Weak stationarity (as defined in Sect. 4). The reason is that, as when time is advanced, the weight on the remaining elements of the stream assigned by the TDU part of $w^{C}$ increases, while the weight on the limit is not affected. Therefore, it is not the case that there exists $a \in[0,1]$ such that, for all $\mathbf{x}, \mathbf{y} \in \mathbf{X}^{c}$ with $x_{1}=y_{1}=a, w^{C}(\mathbf{x}) \geq w^{C}(\mathbf{y})$ if and only if $w^{C}\left(\mathbf{x}^{\prime}\right) \geq w^{C}\left(\mathbf{y}^{\prime}\right)$, where $x_{t}^{\prime}=x_{t+1}$ and $y_{t}^{\prime}=y_{t+1}$ for all $t \in \mathbb{N}$. Rather, for all $a \in[0,1]$, there exist $\mathbf{x}, \mathbf{y} \in \mathbf{X}^{c}$ with $x_{1}=y_{1}=a$, satisfying

$$
w_{\beta}^{T}(\mathbf{x})>w_{\beta}^{T}(\mathbf{y}) \text { and } \lim _{t \rightarrow \infty} x_{t}<\lim _{t \rightarrow \infty} y_{t},
$$

such that $w^{C}(\mathbf{x})<w^{C}(\mathbf{y})$, but $w^{C}\left(\mathbf{x}^{\prime}\right)>w^{C}\left(\mathbf{y}^{\prime}\right)$, where $x_{t}^{\prime}=x_{t+1}$ and $y_{t}^{\prime}=y_{t+1}$ for all $t \in \mathbb{N}$. In particular, if $\mathbf{x}=\left(a, 1,1,0_{\text {con }}\right)$ and $\mathbf{y}=\left(a, 0,0,1_{\text {con }}\right)$ with $\beta=1 / 2$, $\gamma=1 / 4$ and $u(x)=x$, then $w_{\beta}^{T}\left(\mathbf{x}^{\prime}\right)=3 / 4$ and $w_{\beta}^{T}\left(\mathbf{y}^{\prime}\right)=1 / 4$ so that $w^{C}\left(\mathbf{x}^{\prime}\right)-$ $w^{C}\left(\mathbf{y}^{\prime}\right)=1 / 8$ and $w^{C}(\mathbf{x})-w^{C}(\mathbf{y})=-1 / 16$ for all $a \in[0,1]$.

In contrast, the RDU SWF $w_{\beta}^{R}$ satisfies Weak stationarity since, for all $\mathbf{x}, \mathbf{y} \in \mathbf{X}$ with $x_{1}=y_{1}=0($ or $=1), w_{\beta}^{R}(\mathbf{x}) \geq w_{\beta}^{R}(\mathbf{y})$ if and only if $w_{\beta}^{R}\left(\mathbf{x}^{\prime}\right) \geq w_{\beta}^{R}\left(\mathbf{y}^{\prime}\right)$, where $x_{t}^{\prime}=x_{t+1}$ and $y_{t}^{\prime}=y_{t+1}$ for all $t \in \mathbb{N}$. The key is that, by setting $a=0$ (or $=1$ ), the internal ranking of the elements of $\mathbf{x}^{\prime}$ and $\mathbf{y}^{\prime}$ remains unchanged when the wellbeing level $a=0$ (or $=1$ ) is imposed at time 1 and the elements of $\mathbf{x}^{\prime}$ and $\mathbf{y}^{\prime}$ are moved one period forward. However, $w_{\beta}^{R}$ fails Separable future. Indeed:

$$
\begin{aligned}
\mathbf{x} & =\left(\frac{2}{5}, \frac{2}{5}, \frac{2}{5} \text { con }\right) & \mathbf{y} & =\left(\frac{2}{5}, 0,1_{\text {con }}\right) \\
\mathbf{x}^{\prime} & =\left(0, \frac{2}{5}, \frac{2}{5} \text { con }\right) & \mathbf{y}^{\prime} & =\left(0,0,1_{\text {con }}\right)
\end{aligned}
$$

with $\beta=1 / 2$ and $u(x)=x$, then $w_{\beta}^{R}(\mathbf{x})=8 / 20>7 / 20=w_{\beta}^{R}(\mathbf{y})$, while $w_{\beta}^{R}\left(\mathbf{x}^{\prime}\right)=$ $4 / 20<5 / 20=w_{\beta}^{R}\left(\mathbf{y}^{\prime}\right)$, thereby contradicting Separable future. As a result, also

\footnotetext{
7 See also Mitra and Ozbek (2013) for further results on the representability of infinite wellbeing streams.
} 
Stationarity is contradicted, because $w_{\beta}^{R}\left(\mathbf{x}^{\prime \prime}\right)=4 / 10<5 / 10=w_{\beta}^{R}\left(\mathbf{y}^{\prime \prime}\right)$, where $x_{t}^{\prime \prime}=x_{t+1}$ and $y_{t}^{\prime \prime}=y_{t+1}$ for all $t \in \mathbb{N}$.

\section{References}

Alcantud, J.C.R.: Inequality averse criteria for evaluating infinite utility streams: The impossibility of Weak Pareto. J. Econ. Theory 147, 353-363 (2012)

Asheim, G.B.: Individual and collective time-consistency. Rev. Econ. Stud. 64, 427-443 (1997)

Asheim, G.B., Ekeland, I.: Resource conservation across generations in a Ramsey-Chichilnisky model. Econ. Theory 61, 611-639 (2016)

Asheim, G.B., Mitra, T.: Sustainability and discounted utilitarianism in models of economic growth. Math. Soc. Sci. 59, 148-169 (2010)

Banerjee, K., Mitra, T.: On Wold's approach to representation of preferences. J. Math. Econ. 79, 65-74 (2018)

Basu, K., Mitra, T.: Aggregating infinite utility streams with intergenerational equity: the impossibility of being Paretian. Econometrica 71, 1557-1563 (2003)

Basu, K., Mitra, T.: Utilitarianism for infinite utility streams: A new welfare criterion and its axiomatic characterization. J. Econ. Theory 133, 350-373 (2007)

Beals, R., Koopmans, T.C.: Maximizing stationary utility in a constant technology. SIAM J. Appl. Math. 17, 1001-1015 (1969)

Blackorby, C., Nissen, D., Primont, D., Russell, R.R.: Consistent intertemporal decision making. Rev. Econ. Stud. 40, 239-248 (1973)

Bossert, W., Sprumont, Y., Suzumura, K.: Ordering infinite utility streams. J. Econ. Theory 135, 579-589 (2007)

Chambers, C.: Intergenerational equity: sup, inf, lim sup, and lim inf. Soc. Choice Welfare 32, 243-252 (2009)

Chichilnisky, G.: An axiomatic approach to sustainable development. Soc. Choice Welfare 13, 231-257 (1996)

Crespo, J.A., Nuñez, C., Rincón-Zapatero, J.P.: On the impossibility of representing infinite utility streams. Econ. Theory 40, 47-56 (2009)

Diamond, P.: The evaluation of infinite utility streams. Econometrica 33, 170-177 (1965)

Dockner, E., Wagener, F.: Markov perfect Nash equilibria in models with a single capital stock. Econ. Theory 56, 585-625 (2014)

Dutta, B.: Some remarks on the ranking of infinite utility streams. In: Basu, K., Kanbur, R. (eds.) Arguments for a Better World: Essays in Honor of Amartya Sen, Volume I: Ethics, Welfare and Measurement, pp. 136-147. Oxford University Press, Oxford (2008)

Fleurbaey, M., Michel, P.: Intertemporal equity and the extension of the Ramsey criterion. J. Math. Econ. 39, 777-802 (2003)

Halevy, Y.: Time consistency: stationarity and time invariance. Econometrica 83, 335-352 (2015)

Jackson, M.O., Yariv, P.: Collective dynamic choice: The necessity of time inconsistency. AEJ: Microecon 7, 150-178 (2015)

Jonsson, A., Voorneveld, M.: The limit of discounted utilitarianism. Luleå University of Technology and Stockholm School of Economics, Mimeo (2015)

Jonsson, A., Voorneveld, M.: The limit of discounted utilitarianism. Theor. Econ. 13, 19-37 (2018)

Koopmans, T.C.: Stationary ordinal utility and impatience. Econometrica 28, 287-309 (1960)

Lauwers, L.: Ordering infinite utility streams comes at the cost of a non-Ramsey set. J. Math. Econ. 46, 32-37 (2010)

Millner, A., Heal, G.: Time consistency and time invariance in collective intertemporal choice. J. Econ. Theory 176, 158-169 (2018)

Mitra, T., Ozbek, M.K.: On representation of monotone preference orders in a sequence space. Soc. Choice Welfare 41, 473-487 (2013)

Mitra, T., Sorger, G.: Extinction in common property resource models: an analytically tractable example. Econ. Theory 57, 41-57 (2014)

Pollak, R.A.: Consistent planning. Rev. Econ. Stud. 35, 201-208 (1968)

Ramsey, F.: A mathematical theory of saving. Econ. J. 38, 543-59 (1928) 
Sakai, T.: Limit representations of intergenerational equity. Soc. Choice Welfare 47, 481-500 (2016)

Sorger, G.: Markov-perfect Nash equilibria in a class of resource games. Econ. Theory 11, 79-100 (1998)

Zame, W.R.: Can intergenerational equity be operationalized? Theor. Econ. 2, 187-202 (2007)

Zuber, S., Asheim, G.B.: Justifying social discounting: the Rank-discounted utilitarian approach. J. Econ. Theory 147, 1572-1601 (2012)

Publisher's Note Springer Nature remains neutral with regard to jurisdictional claims in published maps and institutional affiliations. 